\title{
Level Set Method Based on Interval Analysis
}

\author{
E. de Weerdt ${ }^{*}$, E.R. van Oort ${ }^{\dagger}$, E. van Kampen ${ }^{\ddagger}$, Q.P. Chu ${ }^{\S}$ \\ Delft University of Technology, Control and Simulation Division \\ P.O. Box 5058, 3600 GB Delft, The Netherlands
}

\begin{abstract}
Level set methods are used to determine the reachable set for a given time domain. In the aerospace industry level set methods are used to determine for example flight envelopes. A new level set method is presented which uses interval analysis to give guaranteed bounds on the solution. Unlike the current grid-based methods the new method does not use a grid and has a lower computation complexity. The guaranteed bounds are a product of using interval analysis. Both an inner and outer bound can be derived. The time step and accuracy are easily controlled by the user and can be automatically adapted during processing. Initial tests show that the new method provides accurate solutions and does so using lower computational loads. The new method is tested on a one-dimensional and a two-dimensional test bed, showing that correct outer bounds of the reachable sets can be computed.
\end{abstract}

\section{Introduction}

Flight safety is of utmost importance for all stakeholders in aviation. A majority (59\%) of (fatal) aircraft accidents in a ten year period from 1997-2006 was associated with Loss-of-Control (LOC). ${ }^{1}$ One definition for LOC is that the (auto)pilot is not able to return to the normal flight envelope from the current flight condition; the Safe Flight Envelope (SFE) is exceeded. Derivation of the SFE is important for all aircraft, since LOC accidents happen to general aviation, military and scheduled transport aircraft. ${ }^{2}$ For undamaged aircraft the SFE can be approximated using flight testings. However, when an aircraft sustains damage during flight, the SFE will generally shrink and/or shift. Knowledge of the SFE is therefore even more important for aircraft with failures, since the performance limits have changed and the guidelines and limits in effect for the nominal aircraft are inadequate.

An effective method to prevent LOC accidents is envelope protection, prohibiting the aircraft from entering potentially dangerous parts of the flight envelope. The SFE can be defined as the set of states that can be reached from the set of all trim states and can also reach a trim, or equilibrium, state within finite time. Determining the SFE is not a trivial problem given the complex nature of aircraft dynamics.

One method of checking safety is by simulation of a single trajectory of the system at a time. However, for systems with many different state values and input signals this would be far too computationally expensive. Even for a few million simulations, important but isolated trajectories that lead to unsafe states can be easily missed. A possible solution for this is the computation of reachable sets. Reachable sets are a way of capturing the behavior of entire groups of trajectories at once. In Ref. 3 a combination of reachability and region of attraction analysis is used to assess the flight envelope for a generic transport aircraft model. Region of attraction is also applied in Ref. 4 by solving a Sum-of-Squares (SOS) relaxation. A popular method to describe a set of continuous states is called the implicit surface representation. Level set methods ${ }^{5}$ are a collection of numerical techniques for evolving implicit surfaces according to underlying flow fields. Their governing equations are Hamilton-Jacobi (HJ) partial differential equations (PDE). Polytopic approximations of the reachable set using level set methods for linear games are presented in Ref. 6. Reachable set theory

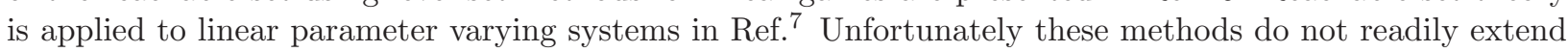

\footnotetext{
*PhD student, student AIAA member

${ }^{\dagger}$ PhD student, student AIAA member

${ }^{\ddagger}$ Assistant professor, AIAA member

$\S$ Associate professor, AIAA member
} 
to general nonlinear systems. More general nonlinear systems are considered by Refs. 8-10 which use level set methods to solve evolution of the reachable set through a HJ PDE. An excellent MATLABC) level set toolbox has been developed by Mitchell. ${ }^{11}$ An inner and outer approximation of capture basins using interval analysis is proposed in Ref. 12. However, as noted by the others, this method requires a large number of bisections for accurate approximations and even more bisections for small target sets.

In this paper an alternative method is presented to compute approximations of the reachable set based on the level set HJ PDE. Using interval arithmetic ${ }^{13}$ this PDE can be evaluated on boxes in the domain, evolving the boundary of reachable set. Using this method, guaranteed over- and under-approximations of the reachable set can be computed for nonlinear systems, not necessarily affine in the input and disturbances. Input and disturbance signals and their constraints are handled inherently by the interval arithmetic. Furthermore the time step and accuracy of the method are easily controlled by the user. Other examples of the application of interval analysis to aerospace related problems are for example: finding trim-points for nonlinear aircraft models ${ }^{14}$ pilot model identification, ${ }^{15}$ integer ambiguity resolution for aircraft attitude determination, ${ }^{16,17}$ spacecraft re-entry optimization ${ }^{18}$ and fuel optimization for constrained spacecraft formation rotations. ${ }^{19}$

This paper is organized as follows. In Section II problem of reachable set determination is reformulated as the solution of a Hamilton-Jacobi-Isaacs PDE. Section III gives a brief introduction and description of interval arithmetic. The new method based on IA to obtain an approximate solution of the problem described in Section II is discussed in Section IV. Numerical results for a number of examples are presented in Section V. Finally, in Section VI conclusions are drawn and future research directions are indicated.

\section{Level set theory}

This section first introduces implicit interface descriptions. Then, using this concept, partial differential equations are derived which evolve the implicit interface in time according to the system dynamics.

\section{A. Implicit Functions}

In one spatial dimension, the real line can be divided into three distinct pieces, using the points at $x=-1$ and $x=1$. Three intervals are defined as $(-\infty,-1),(-1,1)$ and $(1, \infty)$ as three subdomains. The part $\Omega^{-}=(-1,1)$ will be referred to as the inside part of the domain, and $\Omega^{+}=(-\infty,-1) \cup(1, \infty)$ as the outside part. The border between the inside and outside consists of the two points $\partial \Omega=\{-1,1\}$, and is defined as the interface. Thus, in one spatial dimension the inside and outside regions are one-dimensional, while the interface is zero-dimensional. More generally, in $\mathbb{R}^{n}$, subdomains are $n$-dimensional, while the interface has dimension $n-1$.

In an explicit representation of the interface, all segments belonging to the interface are explicitly defined, as in the example in one dimension above. An alternative description is the implicit definition of the interface as an isocontour of some function. For example, the zero isocontour of the function $\phi(x)=|x|-1$ is the set of all points where $\phi(x)=0$. Figure 1 shows this implicit description of the boundary $\partial \Omega$. Note that the function $\phi(x)$ is defined over the whole domain, while the interface, or isocontour, is defined in one dimension lower. An implicit interface representation might seem wasteful, however this representation allows the use of very powerful tools for reachable set computation.

\section{B. Reachable Sets}

Level set methods add dynamics to implicitly defined surface descriptions. Consider a dynamical system

$$
\begin{aligned}
\dot{x}= & f(x(t), u(t), d(t)) \\
& x(0) \in \mathcal{S}_{0}\left(\text { or } x\left(t_{f}\right) \in \mathcal{T}_{0}\right), t \in\left[0, t_{f}\right]
\end{aligned}
$$

where $0 \leq t_{f}<\infty, x \in \mathbb{R}^{n}$ is the state, $u \in U \subset \mathbb{R}^{m}$ is the control input, $d \in D \subset \mathbb{R}^{p}$ is the disturbance input, $\mathcal{S}_{0}=\{x: S(x) \leq 0\}$ is an initial set of states, and $\mathcal{T}=\{x: T(x) \leq 0\}$ is a target set of states. The function $f$ is assumed to be Lipschitz. The spaces of admissible control input and disturbance input trajectories are denoted as the spaces of the piecewise continuous functions $\mathcal{U}=\left\{u(\cdot) \in P C^{0} \mid u(t) \in U, 0 \leq t \leq t_{f}\right\}$, and $\mathcal{D}=\left\{d(\cdot) \in P C^{0} \mid d(t) \in D, 0 \leq t \leq t_{f}\right\}$ respectively. The forwards and backwards reachable sets, illustrated in Fig. 2, of the system (1) are defined as follows. 


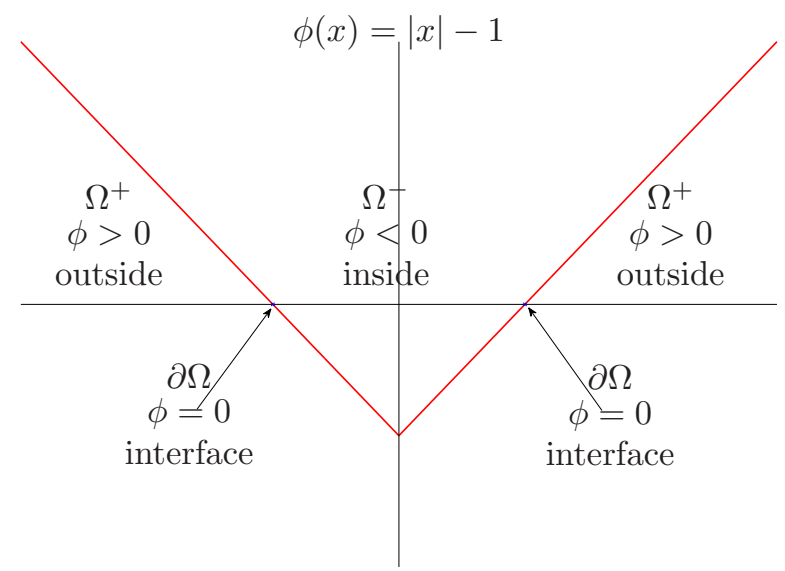

Figure 1. Implicit function $\phi(x)=|x|-1$ defining the region $\Omega^{-}$and $\Omega^{+}$as well as the boundary $\partial \Omega$.

Definition II.1 (Forwards Reachable Set). The forward reachable set $\mathcal{S}(\tau)$ at time $\tau\left(0<\tau \leq t_{f}\right)$ of the system (1) from the initial set $\mathcal{S}_{0}$, is the set of all states $x(\tau)$, such that there exists a control input $u(t) \in \mathcal{U}\left(\tau \leq t \leq t_{f}\right)$, for all disturbance inputs $d(t) \in \mathcal{D}\left(\tau \leq t \leq t_{f}\right)$, for which $x(\tau)$ is reachable from some $x(0) \in \mathcal{S}_{0}$, along a trajectory satisfying (1).

Definition II.2 (Backwards Reachable Set). The backward reachable set $\mathcal{T}(\tau)$ at time $\tau\left(0 \leq \tau \leq t_{f}\right)$, of the system (1) from the target set $\mathcal{T}_{0}$, is the set of all states $x(\tau)$, such that there exists a control input $u(t) \in \mathcal{U}\left(\tau \leq t \leq t_{f}\right)$, for all disturbance inputs $d(t) \in \mathcal{D}\left(\tau \leq t \leq t_{f}\right)$, for which some $x\left(t_{f}\right) \in \mathcal{T}_{0}$ is reachable from $x(\tau)$, along a trajectory satisfying (1).
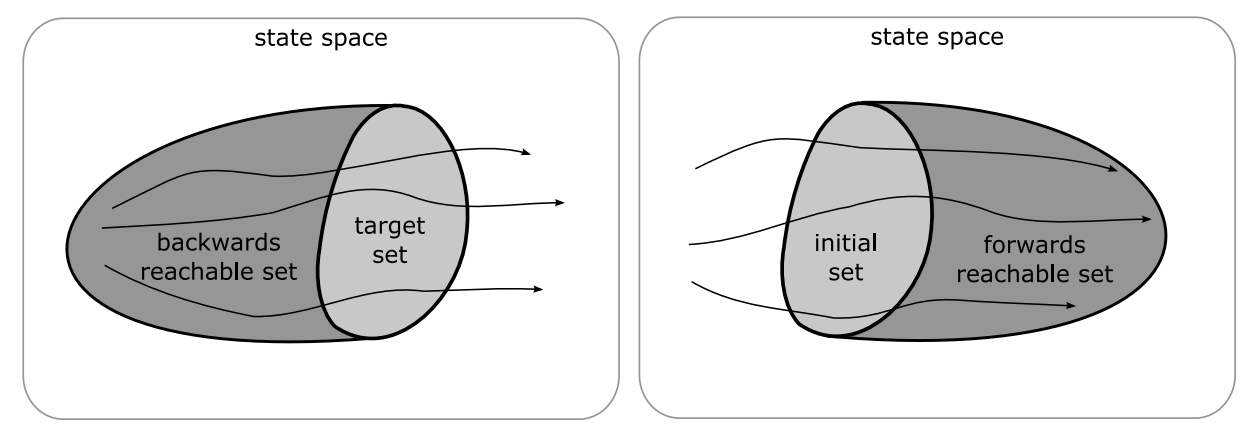

Figure 2. Backwards (left) and forwards (right) reachable set definitions.

It has been shown that there is a connection between the computation of the forwards (backwards) reachable set and a dynamic optimization problem. ${ }^{20,21}$ The forward reachable set of the dynamical system (1) at time $\tau\left(0 \leq \tau \leq t_{f}\right)$ is shown to be ${ }^{20}$

$$
\mathcal{S}(\tau)=\left\{x: \phi_{f}(x, \tau) \leq 0\right\}
$$

where $\phi_{f}(x, \tau)$ is a viscosity solution of the Hamilton-Jacobi-Isaacs (HJI) partial differential equation

$$
\frac{\partial \phi_{f}(x, t)}{\partial t}+\max _{u \in U} \min _{d \in D}\left(\frac{\partial \phi_{f}(x, t)}{\partial x}\right)^{T} f(x, u, d)=0
$$

with $\phi(x, 0)=S(x)$. Therefore, the forward reachable set of the system (1) is the zero sublevel set of the solution to the HJI equation (3). Similarly the backwards reachable set is defined as the zero sublevel set at 
time $\tau\left(0 \leq \tau \leq t_{b}\right)$ of the solution $\phi_{b}(x, t)$ of the HJI equation ${ }^{20}$

$$
\frac{\partial \phi_{b}(x, t)}{\partial t}+\min _{u \in U} \max _{d \in D}\left(\frac{\partial \phi_{b}(x, t)}{\partial x}\right)^{T} f(x, u, d)=0
$$

Using the forwards reachable set $\mathcal{S}\left(t_{f}\right)$ and backwards reachable sets $\mathcal{T}\left(t_{b}\right)$, a safe maneuvering set can be defined as the set $\mathcal{O}=\mathcal{S}\left(t_{f}\right) \cap \mathcal{T}\left(t_{b}\right)=\left\{x: \max \left[\phi_{f}\left(x, t_{f}\right), \phi_{b}\left(x, t_{b}\right)\right] \leq 0\right\}$. This set is illustrated in Fig. 3 . This set represents the part of the state space which can be reached from a given initial set, and also can return to this initial set. In the case of flight envelope protection, one can think of the initial/target set as a given trim point, or the set of trim conditions.

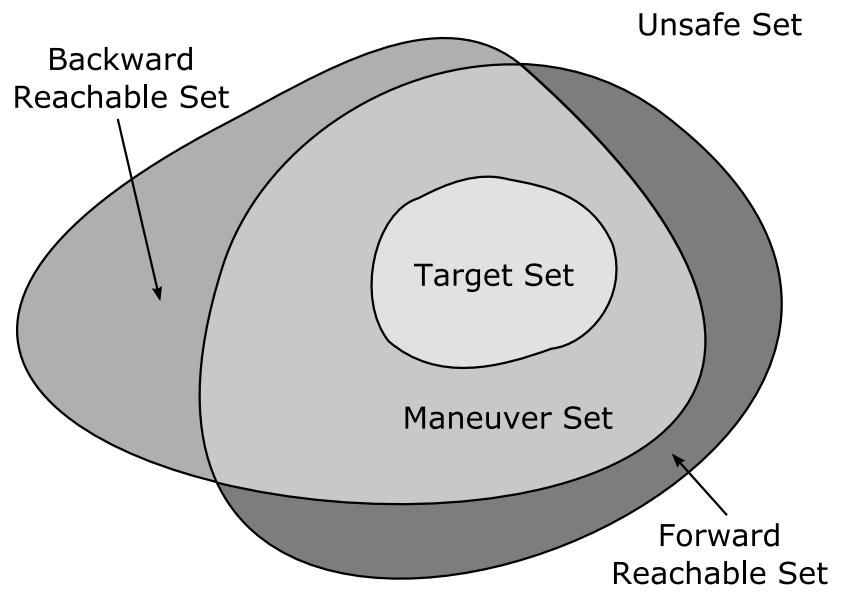

Figure 3. The safe maneuvering set is defined as the intersection between the forwards and backwards reachable set for a given target set.

\section{Interval analysis}

Interval analysis is the theory dealing with interval numbers and the arithmetic operations on them ${ }^{[13,22]}$. The collection of all arithmetic operations on interval numbers is called interval arithmetic. An interval number is defined as an ordered pair of real numbers $[a, b]$ with $a \leq b$. An interval parameter is written with brackets within which either the infimum and supremum are given $[a, b]$ or a single variable $[x]=[a, b]$. Interval arithmetic contains the same operands as ordinary arithmetic such as the basic computational operations of addition, subtraction, multiplication and division:

$$
\begin{aligned}
{[a, b]+[c, d] } & =[a+c, b+d] \\
{[a, b]-[c, d] } & =[a-d, b-c] \\
{[a, b] \cdot[c, d] } & =[\min (a c, a d, b c, b d), \max (a c, a d, b c, b d)] \\
\frac{[a, b]}{[c, d]} & =[a, b] \cdot[1 / d, 1 / c] \quad \text { if } 0 \notin[c, d]
\end{aligned}
$$

The core of interval analysis is to use interval arithmetic to form an inclusion function $[f([\mathbf{x}])]$ of any function $f(\mathbf{x})$. This property of interval arithmetic follows from the inclusion function theorem given by R.E. Moore ${ }^{[13,23]}$ :

Theorem III.1.

If $\left[f\left(\left[x_{1}\right],\left[x_{2}\right], \ldots,\left[x_{n}\right]\right)\right]$ is a rational expression in the interval variables $\left[x_{1}\right],\left[x_{2}\right], \ldots,\left[x_{n}\right]$, i.e. a finite combination of $\left[x_{1}\right],\left[x_{2}\right], \ldots,\left[x_{n}\right]$ and a finite set of constant intervals with interval arithmetic operations, then

implies

$$
\left[x_{1}\right]^{\prime} \subset\left[x_{1}\right],\left[x_{2}\right]^{\prime} \subset\left[x_{2}\right], \ldots,\left[x_{n}\right]^{\prime} \subset\left[x_{n}\right]
$$

$$
\left[f\left([x]_{1}^{\prime},[x]_{2}^{\prime}, \ldots,[x]_{n}^{\prime}\right)\right] \subset\left[f\left(\left[x_{1}\right],\left[x_{2}\right], \ldots,\left[x_{n}\right]\right)\right]
$$


for every set of interval numbers $\left[x_{1}\right],\left[x_{2}\right], \ldots,\left[x_{n}\right]$ for which the interval arithmetic operations in $[f]$ are defined.

Proof. For the proof of this theorem the reader is directed to ${ }^{[23]}$.

If we take $[x]_{1}^{\prime},[x]_{2}^{\prime}, \ldots,[x]_{n}^{\prime}$ to be the crisp numbers $x_{1}, x_{2}, \ldots, x_{n}$ and apply the theorem, then we obtain:

$$
f\left(x_{1}, x_{2}, \ldots, x_{n}\right) \subset\left[f\left(\left[x_{1}\right],\left[x_{2}\right], \ldots,\left[x_{n}\right]\right)\right]
$$

for $x_{1} \subset\left[x_{1}\right], x_{2} \subset\left[x_{2}\right], \ldots, x_{n} \subset\left[x_{n}\right]$. It states that if the input variables lie within the corresponding intervals, interval arithmetic can be used to produce guaranteed bounds on the crisp function output $f(\mathbf{x}) \forall \mathbf{x} \in[\mathbf{x}]$.

An important aspect is the following: if $f(x)$ is a real rational expression in which each variable $x_{i}$ occurs only once and only to the first power, then the function evaluation with interval variables bounds the function $f(x)$ tightly over the set of intervals $\left[x_{i}\right]$, i.e. $\sup [f([x])]=\max _{x \in[x]} f(x)$ and $\inf [f([x])]=\min _{x \in[x]} f(x)$. This is a direct consequence of the properties of interval arithmetic. However, when an interval parameter occurs more than once the bounds on the function output can become non-tight, i.e. $\sup [f([\mathbf{x}])]>\max _{\mathbf{x}} f(\mathbf{x})$ and $\inf [f([\mathbf{x}])]<\min _{\mathbf{x}} f(\mathbf{x}) \forall \mathbf{x} \in[\mathbf{x}]$.

\section{Interval analysis based level set method}

Whenever a new method is being developed it should have advantages over existing methods. To this end, the goal in this paper is to develop a level set method which can provide guaranteed bounds on the reachable set. Moreover, the method must be easy to implement and be applicable to higher dimensional, non-linear, and non-affine problems. Using the inclusion theorem given in the previous section, one can produce guaranteed bounds on the solution as will be shown in this section. To keep the complexity of the method low, the level set function itself is approximated using a linear interpolation technique. In the following section, the new method is given for the case of a one-dimensional problem. First, the method for determining the guaranteed outer bound on the true solution is given (section A). Thereafter, in section B, the method for determining a guaranteed inner bound of the solution is presented. The proposed method is applicable to non-linear non-affine systems. The ideas and aspects presented for the one-dimensional problems are used to construct a solver for higher dimensional problems. First the two-dimensional problem (section C) is discussed before presenting the solver for n-dimensional problems (section D). The discussions in this section concern forward reachable sets. The case of backwards reachable sets is trivial using the same method proposed in this section.

\section{A. Guaranteed outer bound}

As given in section II, the forward reachable set of the dynamical system (1) at time $\tau\left(0 \leq \tau \leq t_{f}\right)$ is shown to be

$$
\mathcal{S}(\tau)=\left\{x: \phi_{f}(x, \tau) \leq 0\right\} .
$$

where $\phi_{f}(x, \tau)$ is a viscosity solution of the Hamilton-Jacobi-Isaacs (HJI) partial differential equation

$$
\frac{\partial \phi_{f}(x, t)}{\partial t}+\max _{u \in U} \min _{d \in D}\left(\frac{\partial \phi_{f}(x, t)}{\partial x}\right)^{T} f(x, u, d)=0
$$

The following theorem based on the previous relations and the inclusion theorem shows that a guaranteed outer approximation can be formed.

Theorem IV.1 (Reachable set guaranteed outer bounds).

Given the definition of the reachable set (eq.2), control domain $U$, disturbance domain D, for space domain $[x]$ and time domain $[t]=\left[0, t_{f}\right]$, an inclusion function can be defined for the reachable set $\mathcal{S}(\tau)$ :

$$
\mathcal{S}(\tau) \subseteq[\mathcal{S}(\tau)]=\left\{x: \varphi(x, 0)-\tau \sup \left[\left(\left[\left.\frac{\partial \varphi}{\partial x}\right|_{[x],[t]}\right]\right)^{T}[f([x],[U],[D])]\right] \leq 0\right\} \forall x \in[x], \tau \in[t]
$$

providing guaranteed outer bounds. 
Proof. The function $\varphi(x, \tau)$ has a lower bound which can be derived for domain $x \in[x], t \in[t]$ according to:

$$
\varphi(x, \tau) \geq \varphi(x, 0)+\tau \min _{x \in[x], t \in[t]} \frac{\partial \varphi}{\partial t} \forall x \in[x], t \in[t]
$$

$\varphi(x, \tau)$ is the solution to the Hamilton-Jacobi-Isaacs (HJI) partial differential equation 3. This relation can be used to determine the partial derivative of the function $\varphi(x, \tau)$ with respect to time:

$$
\frac{\partial \varphi}{\partial t}=-\max _{u \in U} \min _{d \in D}\left(\frac{\partial \varphi}{\partial x}\right)^{T} f(x, u, d)
$$

Using the inclusion theorem III.1 one can obtain guaranteed bounds on the partial derivative with respect to time:

$$
\frac{\partial \varphi}{\partial t} \subseteq-\left(\left[\left.\frac{\partial \varphi}{\partial x}\right|_{[x],[t]}\right]\right)^{T}[f([x],[U],[D])] ; \forall x \in[x], t \in[t]
$$

A guaranteed lower value for the partial derivative can thus be set to:

$$
\min _{x \in[x], t \in[t]} \frac{\partial \varphi}{\partial t} \leq-\sup \left[\left(\left[\left.\frac{\partial \varphi}{\partial x}\right|_{[x],[t]}\right]\right)^{T}[f([x],[U],[D])]\right]
$$

Substitution in equation 15 yields the end result.

Theorem IV.1 provides an upper bound on the partial derivative of $\varphi$ with respect to time. This means that any value of this partial derivative below the given upper bound provides a valid outer bound on the reachable set. To have a unique solution one must add a constraint or cost function which must be selected such that the estimated outer bound on the reachable set is as close as possible to the actual bounds of the reachable set. One possibility is to use the following cost function:

$$
J=\iint_{[x]}-\varphi(x, t) d t d x
$$

When trying to minimize the negative volume of the level set estimate one will drive the $\varphi$ values up which effectively minimizes the domain where $\varphi$ is negative. This will minimize the reachable set and together with the given constraint, the optimization scheme which maximizes the cost function will provide a guaranteed outer bound estimate on the reachable set. Other cost function descriptions are possible which might result in a tighter outer bound fit. The latter is subject to further research. For all cost functions it holds that the sharpness of the outer bound depends on the parameterization method.

The approximation of the level set function must be selected such that the directional derivatives and the cost function are easily computed. In light of using interval analysis it is beneficial to have an approximation method which allows the computation of the previous functions without dependency. For these reasons a linear approximation method is selected (see figure 4):

$$
\varphi(\mathbf{x}, t)=c_{0}+\frac{c_{t}}{\Delta t} t+\sum_{i} \frac{c_{i}}{\Delta x_{i}} x_{i}
$$

For this function the directional derivatives are very simple:

$$
\begin{aligned}
\frac{\partial \varphi}{\partial t} & =\frac{c_{t}}{\Delta t} \\
\frac{\partial \varphi}{\partial x_{i}} & =\frac{c_{i}}{\Delta x_{i}}
\end{aligned}
$$

and the cost function becomes:

$$
J=-|\Delta t| \prod_{i}\left(\left|\Delta x_{i}\right|\right)\left(c_{0}+\frac{1}{2} c_{t}+\frac{1}{2} \sum_{i} c_{i}\right)
$$




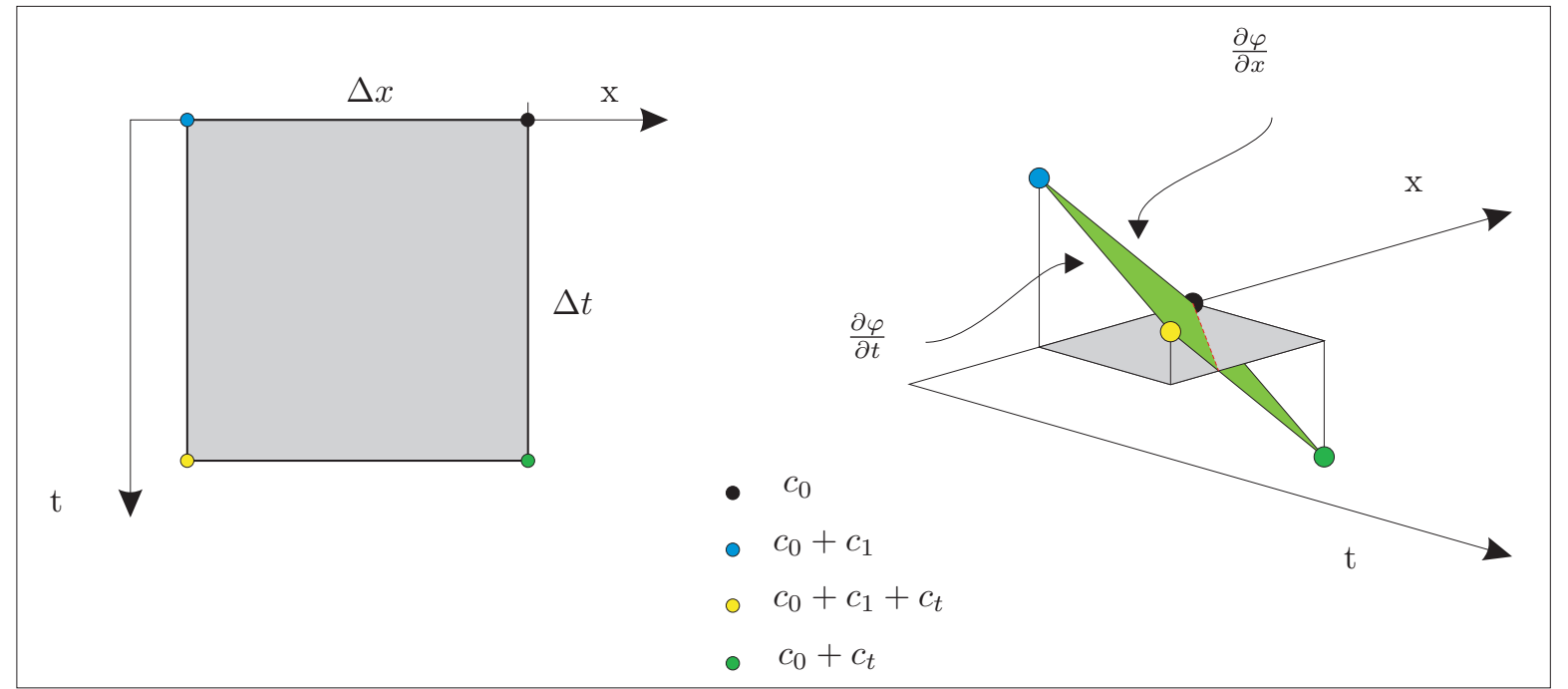

Figure 4. Linear approximation method used for the new level set method - single state example

where $n$ is the problem dimensionality (number of states). Note that in both the derivatives, as in the cost function, the dependent variables occur only once meaning that the obtained bounds are tight (see section III). Moreover, the partial derivatives are constant over the entire domain. This eases the validation of the border constraint (eq 3) and means that the entire surface can be fixed using $(n+2)$ coefficients: $c_{0}, c_{t}, c_{i} \quad i=1,2, \ldots, n$. In the remaining text of this section the principle is explained using the given approximation method and regarding a one-dimensional problem, i.e. $n=1$.

Using the given level set function definition, the partial derivative of $\varphi$ with respect to time becomes:

$$
\frac{\partial \varphi(x, t)}{\partial t}=\frac{c_{t}}{\Delta t} \leq-\frac{c_{i}}{\Delta x_{i}}[f([x],[u])]
$$

where the knowledge of having crisp coefficients and crisp $\Delta t, \Delta x$ is used. There are several observations to be made at this time:

1. $c_{1}+c_{0}$ must be larger than zero otherwise the entire domain $\Delta x$ at $t=0$ is reachable $\varphi<0$.

2. $c_{t}$ must be as large as possible to minimize the cost function

Using the previous conclusions one can rewrite the constraint:

$$
c_{t}=-c_{i} \sup \left[\frac{\Delta t}{\Delta x_{i}}[f([x],[u])]\right]
$$

At this point the sign of $\Delta x$ and $\Delta t$ determine whether the infimum or supremum of $[f]$ must be selected to compute the coefficient value $c_{t}$ :

$$
\begin{aligned}
c_{t} & =-c_{i} \frac{|\Delta t|}{\left|\Delta x_{i}\right|} \sup \left[\frac{\Delta t}{|\Delta t|} \frac{\left|\Delta x_{i}\right|}{\Delta x_{i}}[f([x],[u])]\right] \\
& =-c_{i} \frac{|\Delta t|}{\left|\Delta x_{i}\right|} \sup [\bar{t} \bar{n}[f([x],[u])]]
\end{aligned}
$$

where $\bar{n}$ is equal to the normal vector of the border and $\bar{t}$ is the normalized direction of time $(\bar{t}=1$ for forward in time, $\bar{t}=-1$ for backward in time). Substituting the relation for $c_{t}$ into the cost function:

$$
J=-|\Delta t|\left|\Delta x_{1}\right|\left(c_{0}+\frac{1}{2} c_{1}\left(1-\frac{|\Delta t|}{\left|\Delta x_{1}\right|} \sup (\bar{t} \bar{n}[f([x],[u])])\right)\right)
$$


In the previous relation the value of $c_{0}$ is fixed by the definition of the level set function at $t=0$. Assuming that the domain $(\Delta x, \Delta t)$ is chosen such that the corner lies at the border, the value of $c_{0}$ must be equal to zero. What remains is a cost function which is only dependent on the values of $c_{i}, \Delta t$, and $\Delta x_{1}$ :

$$
J=-\frac{1}{2}|\Delta t|\left|\Delta x_{1}\right| c_{1}\left(1-\frac{|\Delta t|}{\left|\Delta x_{1}\right|} \sup (\bar{t} \bar{n}[f([x],[u])])\right)=-\frac{1}{2}|\Delta t|\left|\Delta x_{1}\right| c_{1} A
$$

There are three test cases:

$A<0$ The cost function is minimized if $c_{1}$ is minimized. Since $c_{1}$ must be positive the optimal value will be zero. As explained before the zero value is not feasible therefore $A$ must be zero or positive

$A=0$ The cost function is zero independent of the choice of $c_{1}$.

$A>0$ The cost function is minimal if $c_{1}$ is maximal. Since no conditions are specified for $c_{1}$ other than being positive, the value can be set to $c_{\max }$ which is defined by the user.

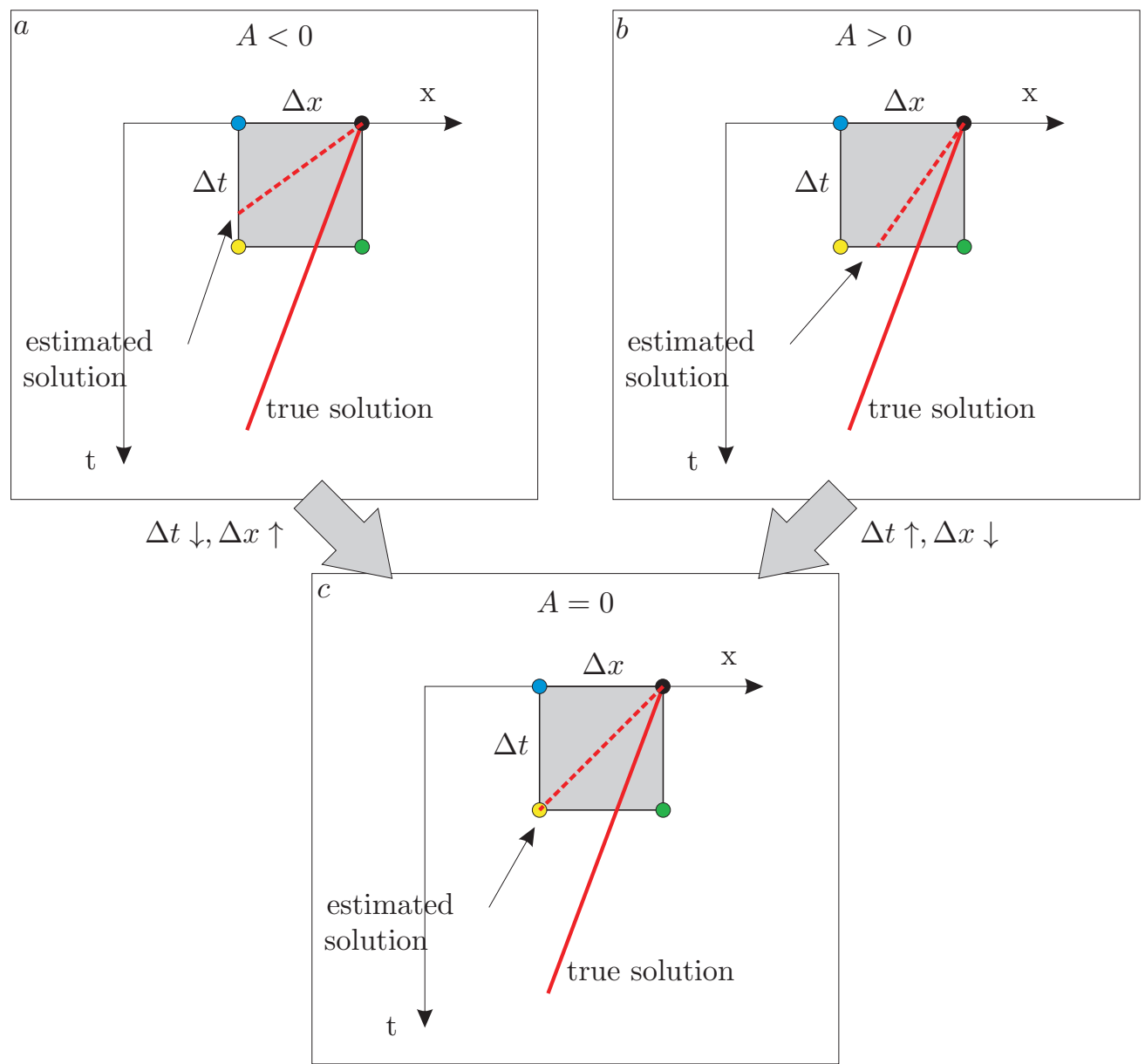

Figure 5. Possible $A$ value and subsequent solutions - single state example

The previous three cases can be graphically represented (see figure 5). If $A>0$ it can have two causes: either

$$
\sup (\bar{t} \bar{n}[f([x],[u])])<0
$$

i.e. cost function is positive regardless of $\Delta x, \Delta t$ values, or the selected $\Delta x$ is too large. The first option means that the border does not grow in the direction of $\Delta x$ but actually shrinks. This means that one can either keep the border stationary or try to compute the retreat of the border. In this paper the assumption is made that the border will always grow or remain stationary. In case the border grows less than $\Delta x$ (option 
two for $A>0$ ) means that the interval $[x]$ used to compute $[f]$ is larger than necessary. If the EOM contain more than one occurrence of $x$ then dependency may be present. Selecting the smallest required $[x]$ may generate tighter bounds for $[f]$. Therefore if $A>0$, then selecting the interval $[x]=x_{1_{0}}+[0, \Delta x]$ (where $x_{1_{0}}$ is the location of the border at $t=0$ ) might yield tighter bounds on $[f]$. If the bounds on $[f]$ are tighter than the growth of the border over the time, then $\Delta t$ will be smaller and closer to the real solution.

If $A<0$ then the selected $\Delta t$ is too large or $\Delta x$ is chosen too small. Either value can be adapted to make $A$ zero. If $\Delta x$ is made larger, then the evaluation of $[f]$ must be performed again. The result might be that the bounds on $[f]$ widen such that for the new $\Delta x$ the value of $A$ is again negative, yielding the same issue. Therefore a safer choice would be to decrease $\Delta t$. If the EOM has a time dependency, then the function $[f]$ has to be reevaluated possibly leading to tighter results (as explained for the case $A>0$ ).

The previous discussion shows that the values of $\Delta t$ and $\Delta x$ can be automatically adapted depending on the value of $A$. This means that the solver can automatically take the largest time step $\Delta t_{\max }$ (set by the user) if the EOM values, i.e. $[f]$, are small and the solver can take smaller steps elsewhere, which is a large advantage of this method. For the one dimensional problems a possible solver for determining a guaranteed outer bound of the solution is given in table 1 . In the next section a solver for the inner bound of the solution is presented.

Table 1. Solver for guaranteed outer bound

0 User defines: border at $t=0: x_{1}^{b}(t=0)$, normal vectors $\bar{n}, \bar{t}$, final time $t_{f}$, maximal time step $\left|\Delta t_{\max }\right|$, maximal domain $\left|\Delta x_{\max }\right|$, control profile $U(t)=\left[u_{\min }(t), u_{\max }(t)\right]$. The value for current time $t_{\text {cur }}$ is set to zero.

1 Define new time domain $[t]=t_{c u r}+\bar{t}\left[0,\left|\Delta t_{\max }\right|\right]$, and $|\Delta t|=\left|\Delta t_{\max }\right|$.

2 Define evaluation box $[x]=x_{1}^{b}\left(t=t_{\text {cur }}\right)+\bar{n}\left[0,\left|\Delta x_{\max }\right|\right]$, and $|\Delta x|=\left|\Delta x_{\max }\right|$.

3 Evaluate the EOM for the current box: $[f([x],[u],[t])]$.

4 Compute the value of $A: A=1-\frac{|\Delta t|}{\left|\Delta x_{1}\right|} \sup (\bar{t} \bar{n}[f])$.

$5 \quad$ IF $(\sup (\bar{t} \bar{n}[f])<0)$ THEN the border cannot grow any further, set border at $t=t_{c u r}+\bar{t}|\Delta t|$ to $x_{1}^{b}\left(t_{c u r}\right)$. If the EOM are time independent, then the border remains equal until the final time, set $|\Delta t|=\left|t_{f}-t_{\text {cur }}\right|$ (no processing needed) GOTO 8 END.

6 IF $A>0$ THEN perform contraction phase (see table 2). GOTO 8 END

$7 \quad$ IF $A<0$ THEN set $|\Delta t|=\frac{|\Delta x|}{\sup (t \bar{n}[f])}$ GOTO 8 END

$8 \quad$ Set $t_{\text {new }}=t_{\text {cur }}+\bar{t}|\Delta t|, x_{1}^{b}\left(t_{\text {new }}\right)=x_{1}^{b}\left(t_{\text {new }}\right)+\bar{n}|\Delta x|$.

$9 \quad \mathrm{IF} t_{\text {new }} \geq t_{f}$ THEN end solver END

10 Set $t_{\text {cur }}=t_{\text {new }}$ GOTO 1 .

Table 2. Contraction phase for outer bound solver

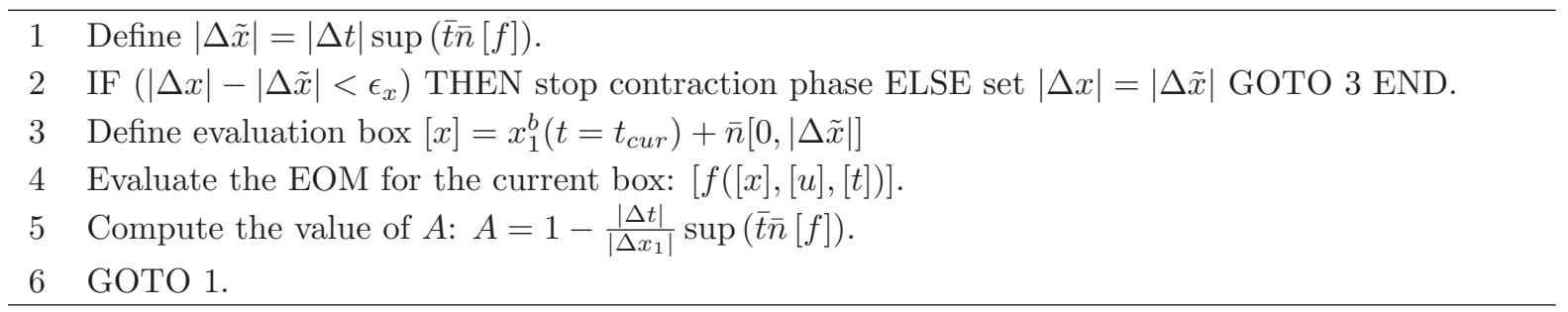

\section{B. Guaranteed inner bound}

The solver for the outer bound approximation is generic in the sense that all types of EOM can be handled. To be able to determine a guaranteed inner bound one has to be able to determine the control parameter values which causes the border to grow at the highest rate. Looking at the border constraint from the theory 
of the level sets:

$$
\frac{\partial \varphi}{\partial t}=-\max _{u} \sum_{i} \frac{\partial \varphi}{\partial x_{i}} f(x, u, t)
$$

one can state that the guaranteed inner bound is obtained when the following constraint is met:

$$
\begin{aligned}
\frac{\partial \varphi}{\partial t} & \geq \sup \left(-\max _{u \in U} \sum_{i} \frac{\partial \varphi}{\partial x_{i}} f([x], u, t)\right) \\
& \geq-\inf \left(\max _{u \in U} \sum_{i} \frac{\partial \varphi}{\partial x_{i}} f([x], u, t)\right)
\end{aligned}
$$

Implementing the latter constraint will ensure, using theorem III.1, that the level set function will be decreased minimally leading to a minimal growth of the reachable set. The problem with the given constraint is that one has to determine the control action which causes the maximal border growth. If the EOM are non-linear this might be difficult.

Similar to the previous section one can use the linear approximation method to obtain a guaranteed inner bound. The cost function however must be inverted to match the goal, i.e. the cost function must resemble the fact that the reachable set must be as large as possible:

$$
J=\int_{[x][t]} \int_{[t]} \varphi d x d t
$$

Substituting the selected approximation method one obtains:

$$
J=|\Delta t| \prod_{i}\left(\left|\Delta x_{i}\right|\right)\left(c_{0}+\frac{1}{2} c_{t}+\frac{1}{2} \sum_{i} c_{i}\right)
$$

Following the same analogy given in the previous section and assuming one-dimensional problems one obtains for the value of $c_{t}$ :

$$
\begin{aligned}
c_{t} & \geq-\inf \left(\max _{u \in U}\left(c_{1} \frac{\Delta t}{\Delta x} f([x], u, t)\right)\right) \\
& \geq-c_{1} \frac{|\Delta t|}{|\Delta x|} \inf \left(\max _{u \in U}(\bar{t} \bar{n} f([x], u, t))\right)
\end{aligned}
$$

The control parameter value which maximizes the given $(\bar{t} \bar{n} f([x], u, t))$ term is denoted $u^{*}$ from this point onward. To minimize the cost function the value of $c_{t}$ must be minimal:

$$
c_{t}=-c_{1} \frac{|\Delta t|}{|\Delta x|} \inf \left(\bar{t} \bar{n} f\left([x], u^{*}, t\right)\right)
$$

Inserting the result in the cost function one obtains:

$$
J=\frac{1}{2}|\Delta t|\left|\Delta x_{1}\right| c_{1}\left(1-\frac{|\Delta t|}{|\Delta x|} \inf \left(\bar{t} \bar{n} f\left([x], u^{*}, t\right)\right)\right)=\frac{1}{2}|\Delta t|\left|\Delta x_{1}\right| c_{1}(-A)
$$

The conclusion regarding the value of $A$ and the subsequent situation as given in figure 5 holds for this case also. The solver for the guaranteed inner bound, see table 3 , is therefore similar to the solver for the outer bounds (table 1 ).

The solver presented in table 3 contains the optimization problem of finding $u^{*}$. A special case for which the optimization problem can easily be solved is when the system is affine in the control, i.e.:

$$
f(x, u, t)=h(x, t)+g(x, t) u
$$

If the EOM are non-affine in the control input, as they in general are, then Taylor Models ${ }^{24-27}$ can be used to transform them to an affine system:

$$
f(x, u, t) \subseteq\left[f\left([x], u_{0},[t]\right)\right]+\left[\left.\frac{\partial f}{\partial u}\right|_{[x][u][t]]}\right]\left(u-u_{0}\right), \forall x \in[x], u \in[u], t \in[t], u_{0} \in[u]
$$


Table 3. Solver for guaranteed inner bound

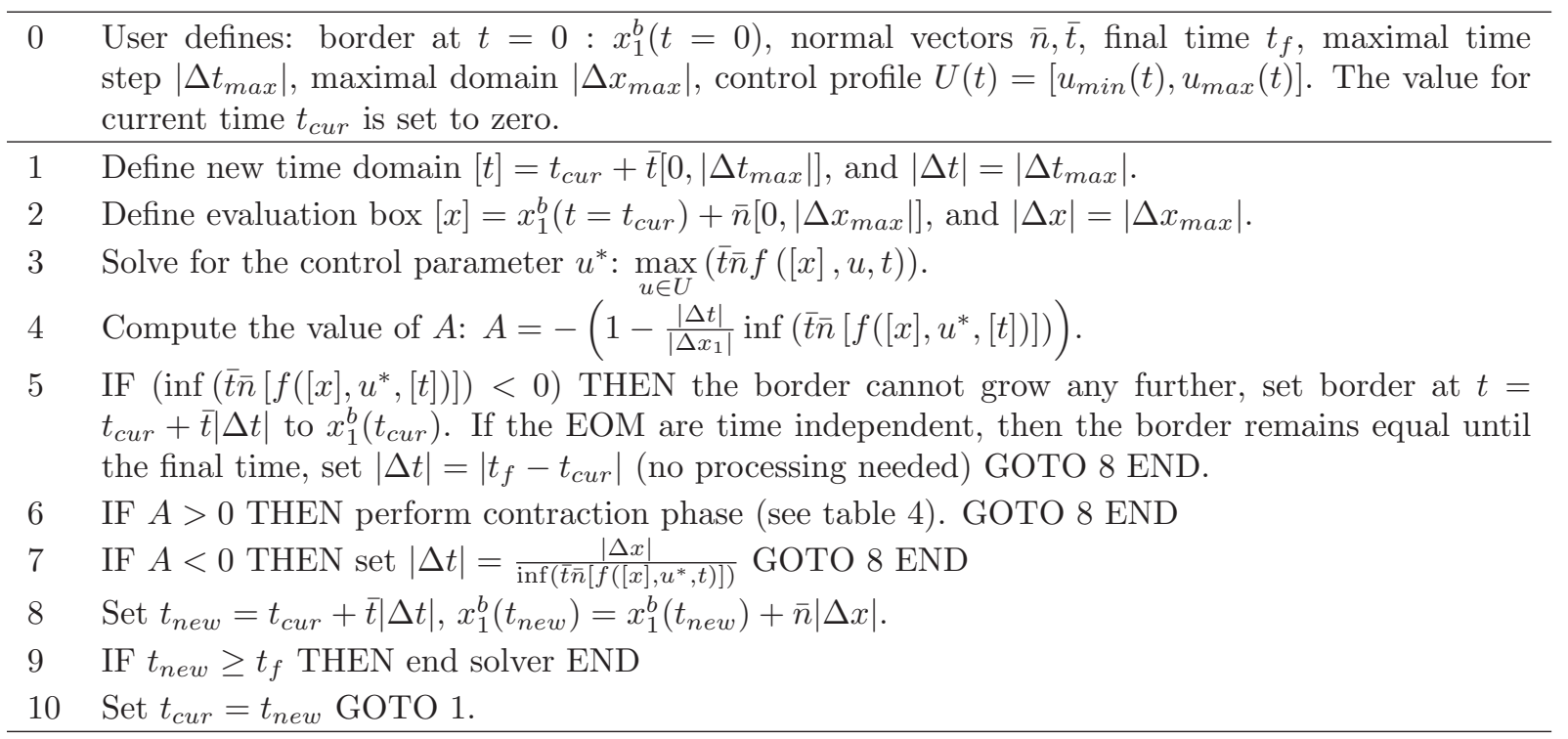

Table 4. Contraction phase for inner bound solver

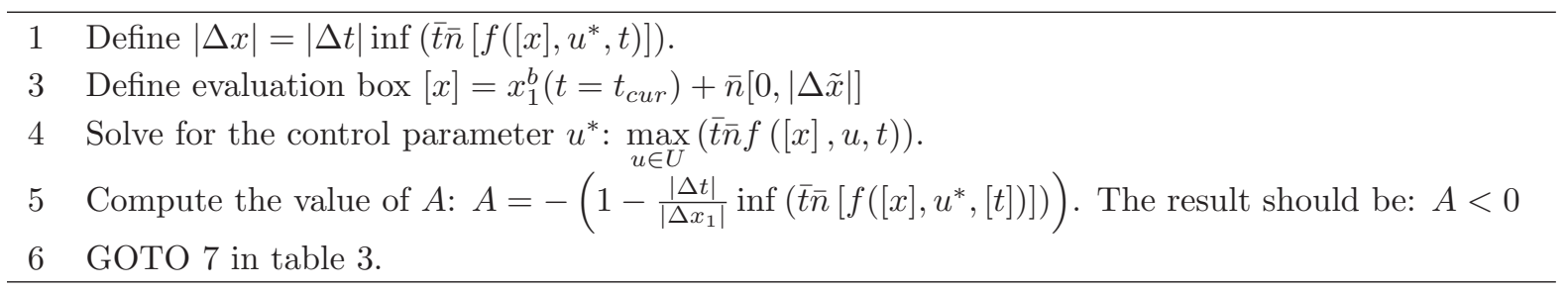


The bounds generated by using a Taylor Model might be non-tight due to dependency and other solvers for finding $u^{*}$ might possibly find tighter bounds. However, the indicated method of using Taylor Model to make the system affine is always applicable. From the previous discussion one can conclude that the proposed method in combination with Taylor Model theory constitutes a generic method applicable to non-linear nonaffine systems. The theory of interval analysis gives the proposed method the capability to always provide a guaranteed outer bound and a guaranteed inner bound for any given problem.

\section{New method applied to two-dimensional problems}

A method is as valuable as its applicability: in theory and in implementation. In the case of level set application, the new method must be applicable to higher dimensional problems. In this section the theory given in the previous section is extended to two-dimensional problems before extending it to general $n$ dimensional problems. Two-dimensional problems are chosen to facilitate the explanation of the method for higher dimensions since the solution can still be graphically represented. The method given in this section is a result of optimizing for ease of implementation and sharpness of guaranteed bounds.

\section{Transformation to the $q$ domain}

The border constraint given in equation 13 can be used to solve for one unknown variable. The cost function itself yields another constraint leading to enough conditions to determine the unknown for two dimensions. When trying to determine the reachable set for higher dimensional problems one has to either add constraints/conditions to solve for the additional unknowns or reduce higher dimensional problems to one-dimensional problems. In this paper the authors have opted for the second approach: reducing dimensionality.

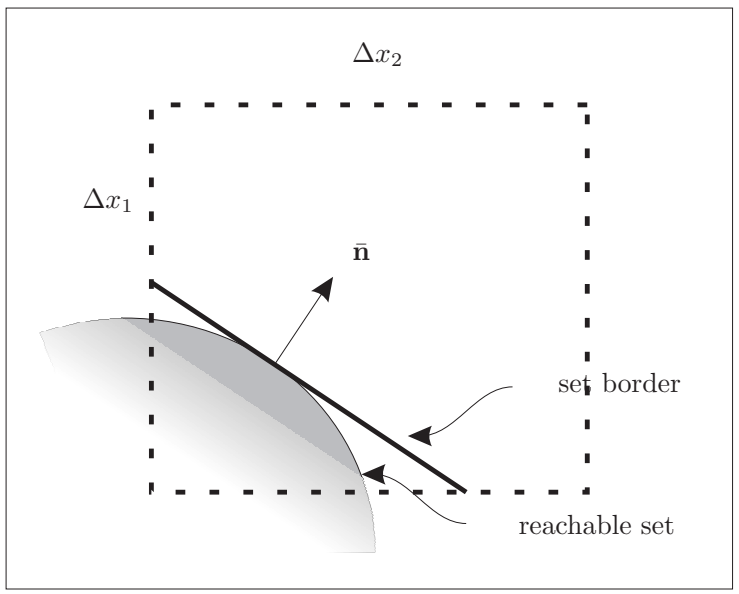

(a) Definition of initial border (outer approximation)

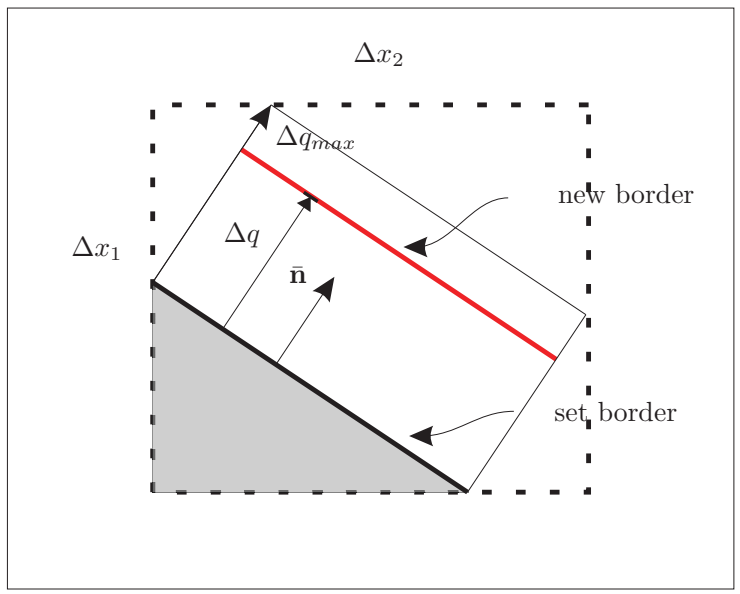

(b) Definition of new border and maximal allowed growth within given box

Figure 6. Example of normal vector $\overline{\mathbf{n}}$ for a reachable set, the definition of the border (old and new), and the maximal allowed growth within a given box $[\mathrm{x}]$.

The idea is to determine the growth of the set border in the direction of the normal vector $\overline{\mathbf{n}}$ (see figure $6(\mathrm{a})$ ). Theorem IV.1 guarantees that the computed border growth in the direction of $\overline{\mathbf{n}}$ is an outer bound on the true solution for any point on the set border. Since points behind the set border cannot grow beyond it, the new border is a guaranteed outer bound for the domain behind the border and within [x] (gray surface in figure $6(\mathrm{~b})$ ). The computation of the border growth in direction of $\overline{\mathbf{n}}$ can be performed by applying the coordinate transformation:

$$
q \overline{\mathbf{n}}=\mathbf{x}
$$

After the coordinate transformation the border growth can be determined in terms of the new coordinate $q$. 
Applying the coordinate transformation to the border constraint one obtains:

$$
\frac{\partial \varphi}{\partial t}+\max _{u \in U} \min _{d \in D} \frac{\partial \varphi}{\partial q} \sum_{i} \overline{\mathbf{n}}_{i} f_{i}(q \overline{\mathbf{n}}, u, t)=0
$$

which constitutes a one-dimensional problem (one state $q$, and time $t$ ) as presented in the previous sections $\mathrm{A}$ and $\mathrm{B}$. The key aspect, which must be applied in order to guarantee the result, is that the final border (computed using $\Delta q$ see figure 6(b)) must lie within the domain of $[x]$ enclosing the initial border and the new border, see figure $6(\mathrm{~b})$. If $\Delta x_{1}, \Delta x_{2}$, and $\overline{\mathbf{n}}$ are given, then the maximal $\Delta q$ can be computed. The final result for the border can be computed using the solvers given in section $\mathrm{A}$ and $\mathrm{B}$.

\section{Guaranteed closed reachable set}

One particularly important aspect is that the border for time $t=t_{0}+\Delta t$, given the border for $t=t_{0}$, must be a closed set. If one implements the method displayed in figure 6 for the entire border starting with a square around the reachable set, then one can end up with the situation represented in figure $7(\mathrm{a})$. The placement and size of the evaluation boxes $[\mathbf{x}]$ is very important. To guarantee that a closed reachable set is found for which the new border is a guaranteed outer approximation, the evaluation boxes $[x]$ must be selected large enough (see figure $7(\mathrm{~b})$ ). If $[x]$ is chosen correctly, then the new borders intersect and the new outer bound estimate can be derived.

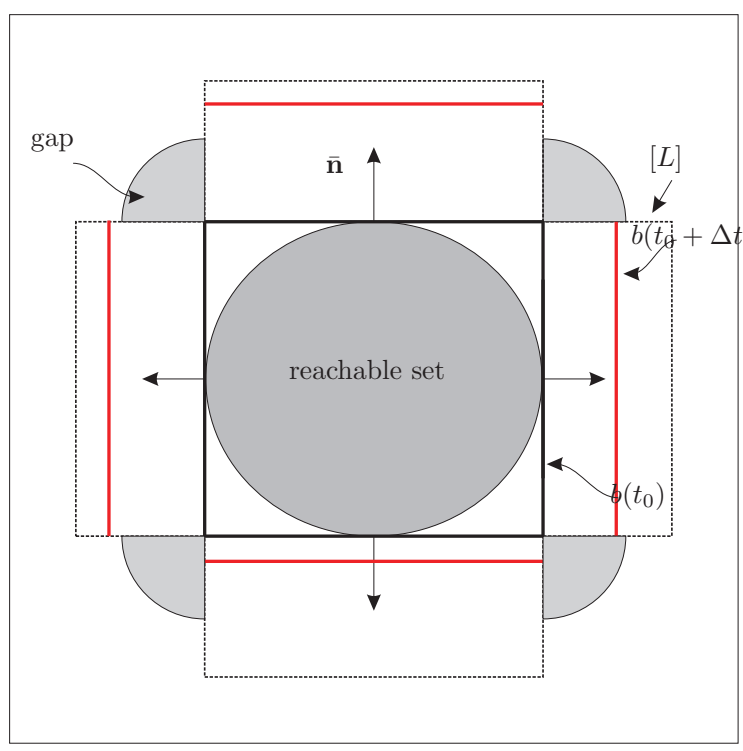

(a) Example of an open reachable set

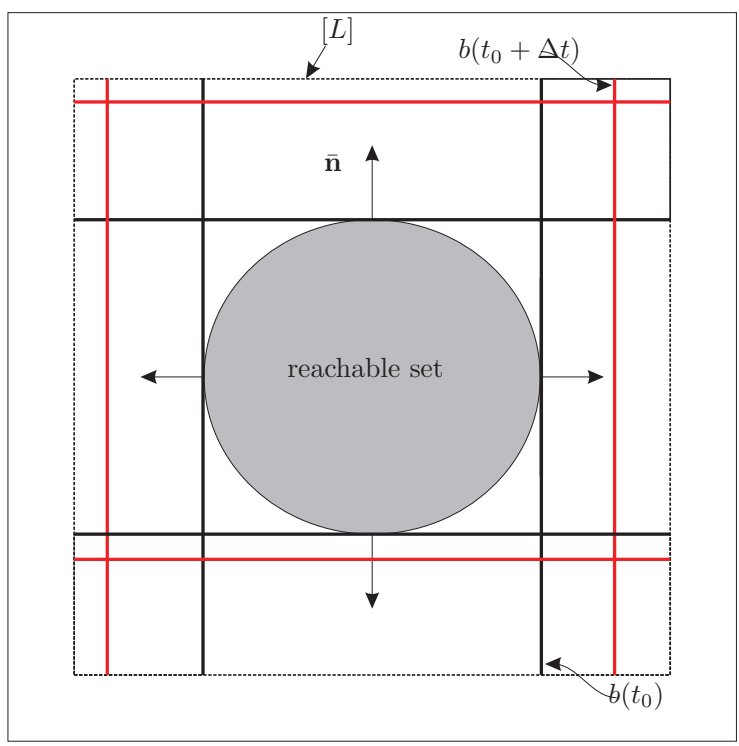

(b) Example of a closed reachable set (dashed blue line)

Figure 7. Example of an open reachable set and a closed reachable set - influence of evaluation box $[\overline{\mathbf{x}}]$ settings.

\section{Solver}

In this paper the approach for solving two-dimensional problems is followed, as presented in figure 8. The user defines the values of $\Delta x_{i}$, and provides the initial set $\mathcal{S}_{0}$ (either as a inequality constraint or a given domain). For the given initial set, one defines the boxes on the border (gray boxes) and which boxes are fully inside the initial set (green boxes). Next comes the propagation step in which the gray boxes are evaluated resulting in the blue domains (the green boxes do not have to be processed). Given the blue domains one can determine which grid cells are fully contained by the estimated outer approximation of the reachable set $\left[\hat{S}\left(t_{0}+\Delta t\right)\right]=[Y]+\left[X\left(t_{0}+\Delta t\right)\right]$. These boxes can be added to the list $[Y]$ and new boxes for the borders are defined (gray boxes) for the next time step.

The given approach is based on rectangles which eases the computation of the normal vectors and subsequent evaluation boxes. Per rectangle $[X]$ there will be $2 * N_{\text {dim }}$ evaluation boxes, 2 evaluation boxes 
per dimension. The first evaluation box for dimension $i$ is given by:

$$
\begin{aligned}
& {\left[L_{j}^{i_{1}}\right]=\left\{\begin{array}{cc}
{\left[\inf \left[X_{j}\right]-\Delta x_{j}, \sup \left[X_{j}\right]+\Delta x_{j}\right]} & \forall j \neq i \\
{\left[\inf \left[X_{j}\right]-\Delta x_{j}, \inf \left[X_{j}\right]\right]} & j=i
\end{array}\right.} \\
& \bar{n}_{j}^{i_{1}} \quad=\left\{\begin{array}{cc}
0 & \forall j \neq i \\
-1 & j=i
\end{array}\right.
\end{aligned}
$$

where the subscript denotes the dimension within box $[L]$ and the subscript $i_{1}$ denotes the first box for dimension $i$. The formulas for the second box for dimension $i$ are:

$$
\begin{aligned}
& {\left[L_{j}^{i_{2}}\right]=\left\{\begin{array}{cc}
{\left[\inf \left[X_{j}\right]-\Delta x_{j}, \sup \left[X_{j}\right]+\Delta x_{j}\right]} & \forall j \neq i \\
{\left[\sup \left[X_{j}\right], \sup \left[X_{j}\right]+\Delta x_{j}\right]} & j=i
\end{array}\right.} \\
& \bar{n}_{j}^{i_{2}}=\left\{\begin{array}{cc}
0 & \forall j \neq i \\
1 & j=i
\end{array}\right.
\end{aligned}
$$

Note that the normal vector is very simple and that the evaluation boxes can thus be easily determined. For a two-dimensional problem one would obtain a total of four evaluation boxes:

$$
\begin{aligned}
{\left[L^{1_{1}}\right] } & =\left[\inf \left[X_{1}\right]-\Delta x_{1}, \inf \left[X_{1}\right]\right],\left[\inf \left[X_{2}\right]-\Delta x_{2}, \sup \left[X_{2}\right]+\Delta x_{2}\right] \\
{\left[L^{1_{2}}\right] } & =\left[\sup \left[X_{1}\right], \sup \left[X_{1}\right]+\Delta x_{1}\right],\left[\inf \left[X_{2}\right]-\Delta x_{2}, \sup \left[X_{2}\right]+\Delta x_{2}\right] \\
{\left[L^{2_{1}}\right] } & =\left[\inf \left[X_{1}\right]-\Delta x_{1}, \sup \left[X_{1}\right]+\Delta x_{1}\right],\left[\inf \left[X_{2}\right]-\Delta x_{2}, \inf \left[X_{2}\right]\right] \\
{\left[L^{2_{2}}\right] } & =\left[\inf \left[X_{1}\right]-\Delta x_{1}, \sup \left[X_{1}\right]+\Delta x_{1}\right],\left[\sup \left[X_{2}\right], \sup \left[X_{2}\right]+\Delta x_{2}\right] \\
n^{1_{1}} & =[-1,0] \\
n^{1_{2}} & =[1,0] \\
n^{2_{1}} & =[0,-1] \\
n^{2_{2}} & =[0,1]
\end{aligned}
$$

corresponding to the situation given in figure 7 (b).

The grid is only used to keep track of the domains which are guaranteed to be included in the reachable set estimate $[\hat{S}]$. Only the boxes containing a part of the border are evaluated making the new method an non-gridded approach. The method uses a list of convex boxes which represent the (possibly) nonconvex reachable set. By using convex boxes the theorem on closed set holds and the new border can be approximated. The grid is used to cut the new boxes if needed and a new list of convex boxes is defined for the next time step.

Given the $\Delta x$ and $\Delta t$ values one can determine (according to solvers for the one-dimensional case, section A) the maximal $\Delta t$ per evaluation box. Note that variable $\Delta t$ steps per evaluation box are permitted and can dramatically decrease the computational load, it is reserved for future research. The individual steps in the solver are given in table 5 . Note that one must be careful to select the proper one dimensional solver. In case that the computed $\Delta q$ is negative then a new evaluation box must be derived (step 6). This evaluation box will have a normal vector directed inward and a new definition of the evaluation box is required. Note that is a box is redefined and reevaluated due to a negative $\Delta q$, then the one-dimensional solver must be inner (outer) bound solver in case one wants to derive and outer (inner) approximation.

\section{N-dimensional problems}

The solver given in the previous section can be easily extended to the $n$-dimensional case. The solver given in table 5 does not change.

\section{Simulation results}

To validate the proposed reachable set approximation scheme, two different examples are presented. The algorithm discussed is implemented in MATLAB(C)2008b, with the use of the INTLAB toolbox for interval computations. ${ }^{28}$ First of all a simple one-dimensional example is considered to show the evolution of the reachable set over time. Secondly, a double integrator example is used. This can be considered as the first 

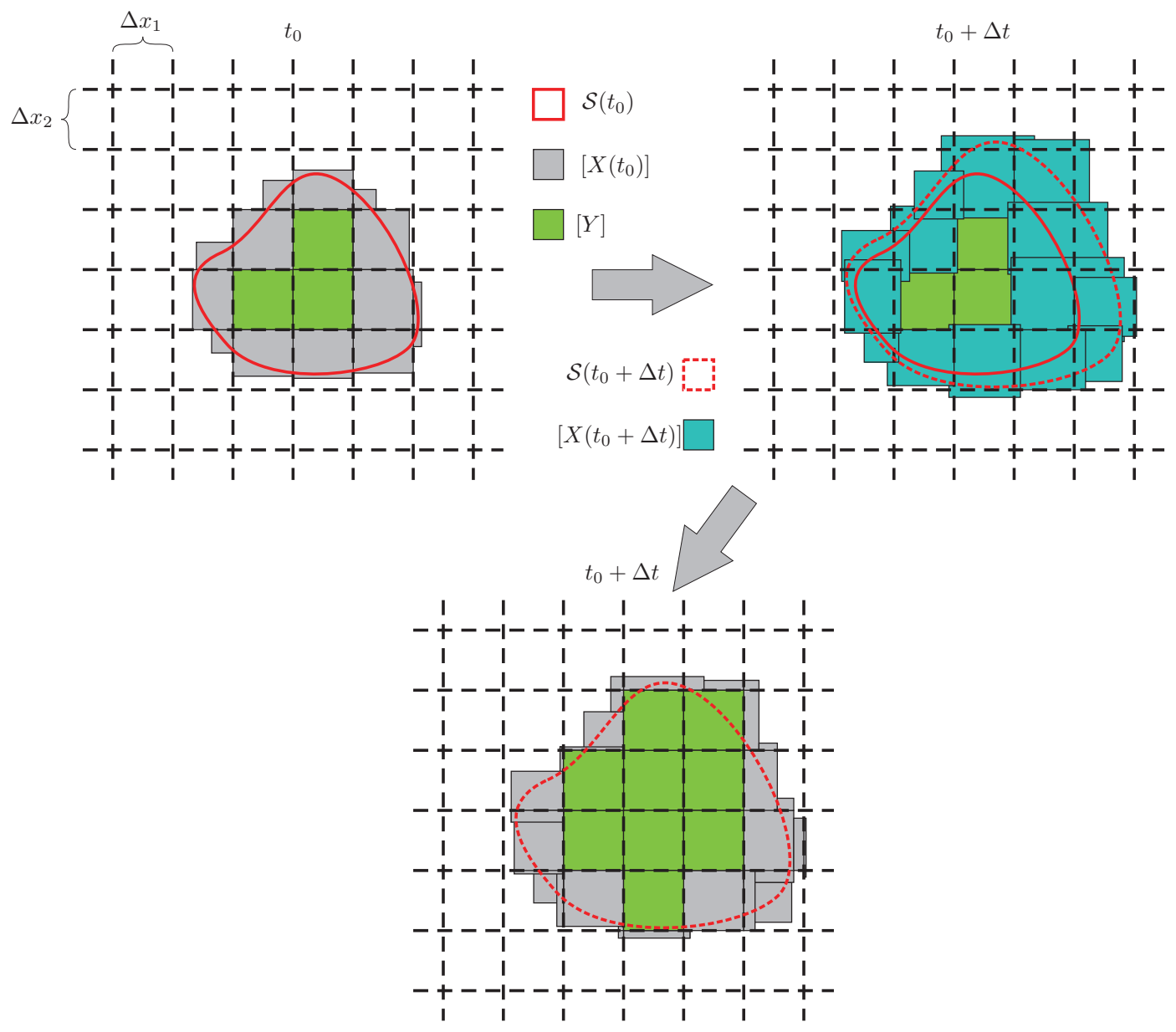

Figure 8. Graphical representation of the evaluation boxes, subsequent growth and reduction of evaluation boxes for the next time step

Table 5. Solver for higher dimensional problems

0 User defines: initial set $\mathcal{S}(0)$, final time $t_{f}$, maximal time step $\left|\Delta t_{\max }\right|$, maximal domain $\left|\Delta x_{\max }\right|$, control profile $U(t)=\left[u_{\min }(t), u_{\max }(t)\right]$. The value for current time $t_{c u r}$ is set to zero.

1 Define grid according to given $\left|\Delta x_{\max }\right|$ per dimension.

2 Given the initial set $\mathcal{S}(0)$, determine the domains containing a part of the border $[X]$ and those fully included in the reachable set $[Y]$.

3 Given the boxes $[X]$, determine the evaluation boxes $[L]$ and the corresponding normal vectors $\overline{\mathbf{n}}$

4 For each of the boxes in $[L]$ determine the maximal time step according to solver 3 , or 1 corresponding to the desire to compute the inner or outer approximation.

5 Take the minimal time step over all boxes. Using this time step, determine the $\Delta q$ per evaluation box $[L]$.

6 IF $\Delta q<0$ THEN redefine the evaluation box and GOTO 4 .

7 If applicable, i.e. $\Delta q<\bar{n} \Delta x_{\max }$, redefine the evaluation boxes $[L]$ and recompute the desired $\Delta q$ using the fixed minimal time step (contraction phase). Perform this contraction phase until decrease in $\Delta q$ is lower than the user defined threshold for all boxes.

8 Using the propagated boxes, determine which domains specified in the grid are fully enclosed $[Y]$ and which contain a part of the new border $[X]$.

9 Set $t_{c u r}=t_{c u r}+\Delta t$ IF $t_{c u r}<t_{f}$ GOTO 3 ELSE end solver. 
real system test since a double integrator can be interpreted as the movement of an object subject to some force. For both examples the analytical reachability solutions are known, therefore the method presented above can be compared with the true reachable set solutions.

\section{A. Low pass filter}

This example is used to show the effect of step size on the outer and inner bounds of the solution. The dynamics of the system are:

$$
\begin{aligned}
& \dot{x}=-x+u \\
& u \in[-0.5,10], S(0):\{|x|<0.01\}, t_{f}=10 s
\end{aligned}
$$

Using the proposed method one obtains the results given in figure 9. The first conclusion is that the bounds correctly enclose the true solution: errors for the inner bounds are always negative and the errors for the outer bounds are always positive. Moreover, one can see that the outer approximation quickly converges to the true solution while the inner approximation requires far smaller $\Delta x$ to reach the same accuracy. The conclusion that the inner bound is more difficult to determine is also valid for higher dimensional problems as will be shown next.

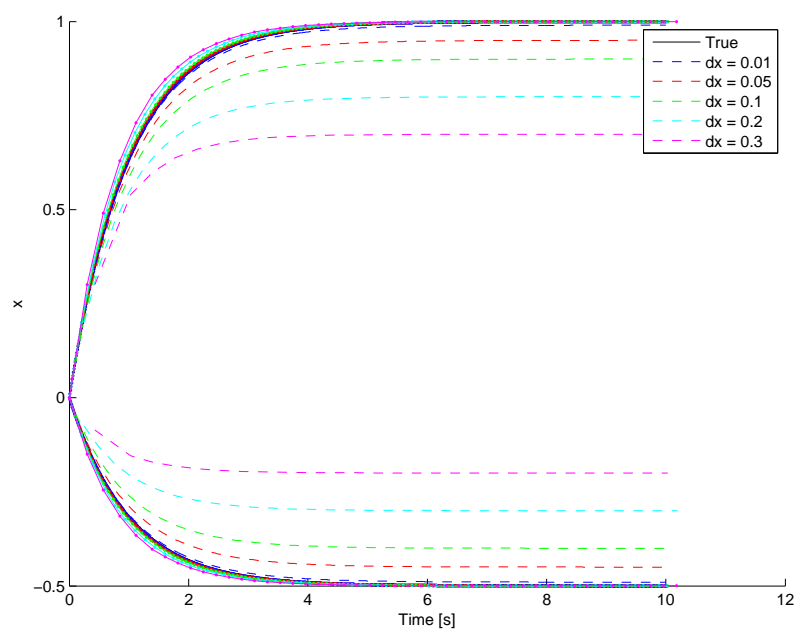

(a) Solutions

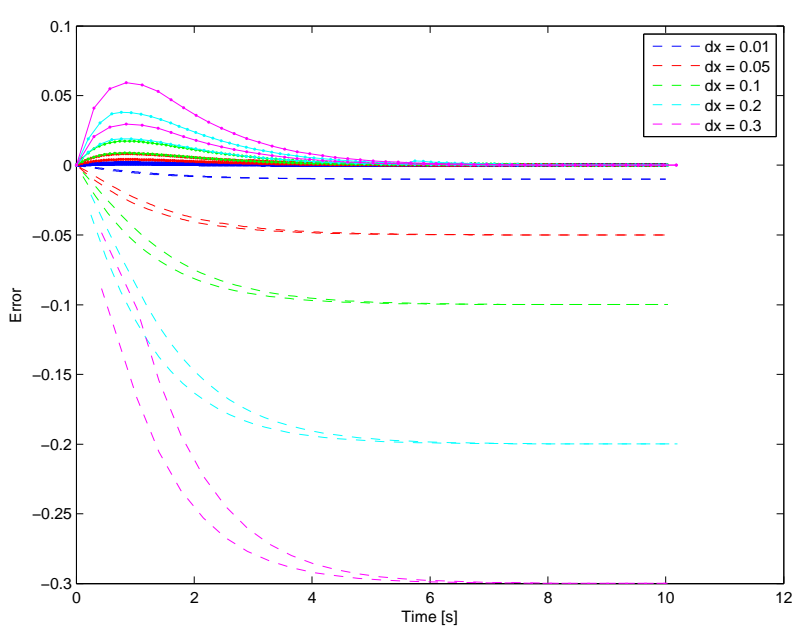

(b) Approximation errors (positive = over-approximation, negative $=$ under-approximation)

Figure 9. Results for experiment 1: one-dimensional test bed. The '.-' lines correspond to the outer bounds and the '-' lines correspond to the inner bounds.

\section{B. Double Integrator}

The double integrator dynamics are written as

$$
\dot{\mathbf{x}}=\left[\begin{array}{c}
x_{2} \\
u
\end{array}\right]=f(\mathbf{x}, u), \quad \mathbf{x}=\left(x_{1}, x_{2}\right)^{T}
$$

where $\mathbf{x} \in \mathbb{R}^{\mathbf{2}}$, and $u \in U$ is the control input. Applying the proposed method to find the outer approximation with the setting $\Delta x_{\max }=0.01$ for both dimensions and $\Delta t_{\max }=1$ (basically letting the dynamics determine the time step using $\left.\Delta x_{\max }\right)$ for time domain $t=[0,1]$ one obtains the results given in figure 10 .

As one can see the outer bound corrected bound the true solution. Especially the outer bound for which the flow $f$ is directed outward (i.e. region $x_{1}<0, x_{2}<0$ and $x_{1}>0, x_{2}>0$ ) are tightly bounded. The plots show that the proposed method can indeed be extended to higher dimensional problems and yield encouraging results. 


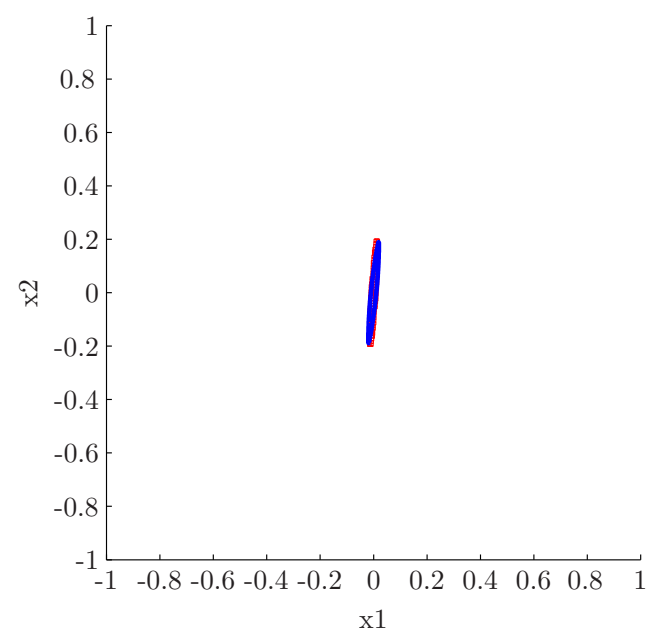

(a) $t=0.2 \mathrm{~s}$

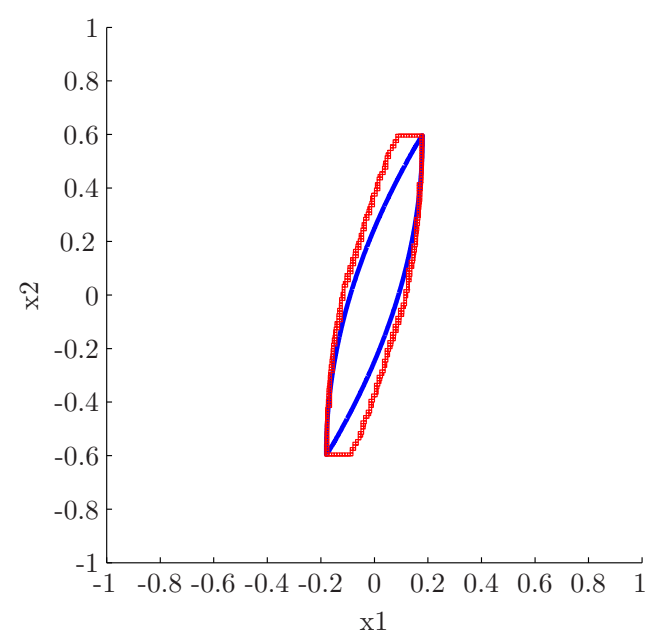

(c) $t=0.6 \mathrm{~s}$

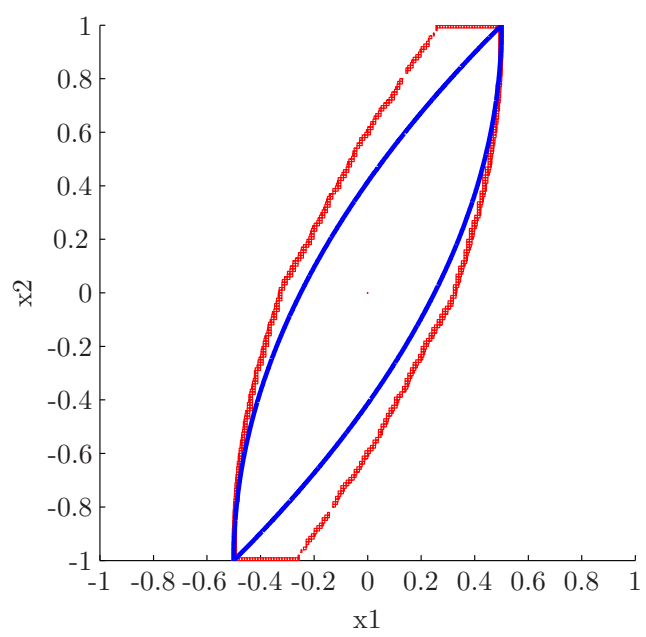

(e) $t=1.0 \mathrm{~s}$

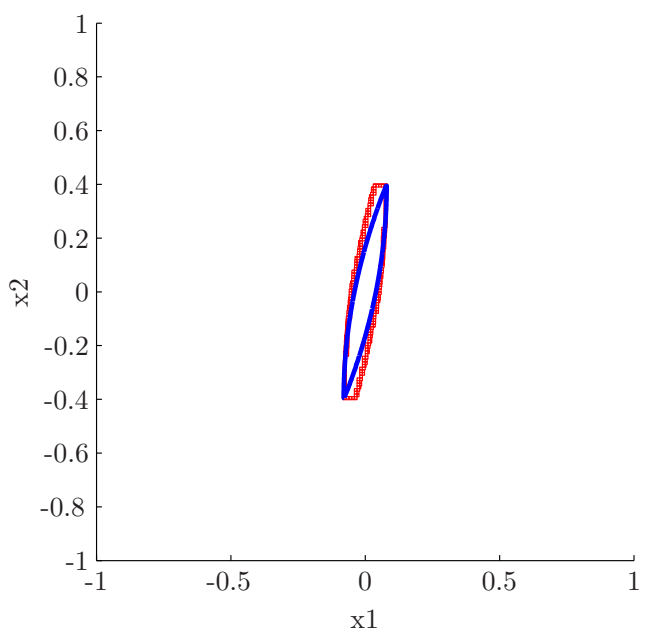

(b) $t=0.4 \mathrm{~s}$

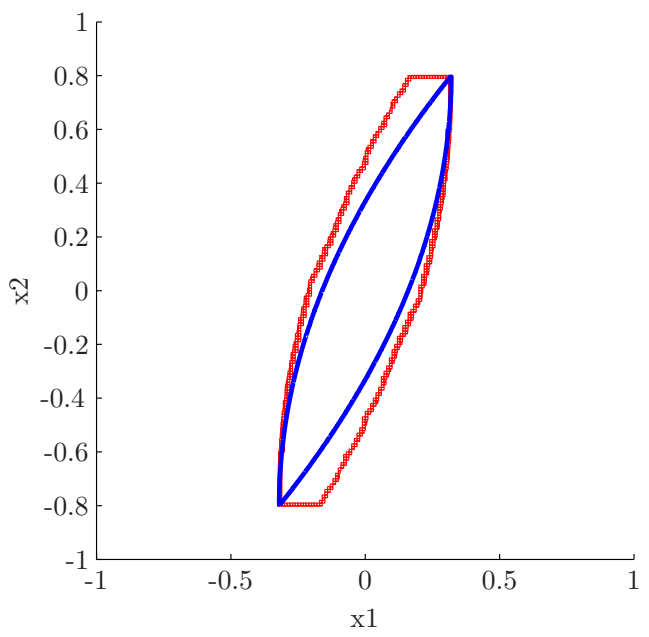

(d) $t=0.8 \mathrm{~s}$

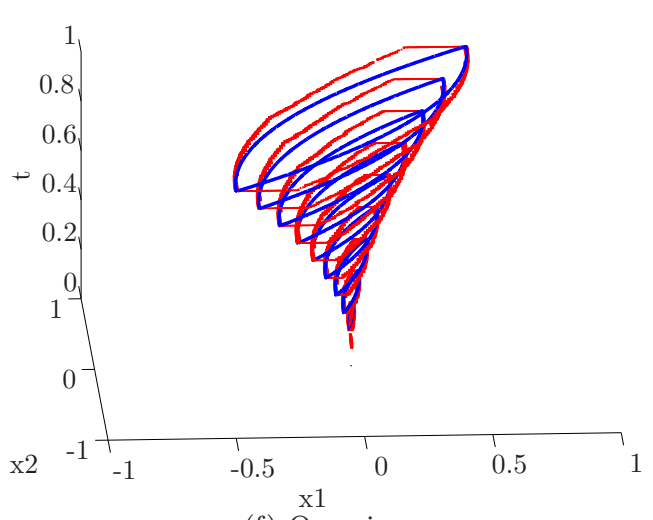

(f) Overview

Figure 10. Outer bounds on the double integrator for $t \in[0,1]$. The blue line represents the analytical solution to the problem and the red boxes denote the guaranteed outer boundary of the level set. 


\section{Conclusions and recommendations}

A new level set method has been introduced which can handle non-linear, non-affine $n$-dimensional problems. Based on the theory of interval analysis, the new method provides a guaranteed inner and outer bound on the reachable set. The performance of the new method has been demonstrated using a one-dimensional and a two-dimensional problem. By applying the method forward and backward in time one can derive a guaranteed Save Envelope within which the system can be driven while still being able to steer the system back to the initial condition. The Safe Envelope is a crucial aspect for flight envelope protection systems, which increase aviation safety.

The new method is unlike any other method. The differences are found in the guaranteed inner and outer bound that other methods cannot provide, and in the method of generating these bounds. The latter is especially important when the new method is compared to other interval analysis based methods. Focus of the comparison lies on the amount of dependency and subsequent sharpness of the derived bounds. The authors believe that the new method derives sharper bounds while having the same computational load.

The proposed method is based on interval analysis and box evaluations. Rectangular boxes have been used since it is easy to determine the required size of the evaluation boxes and subsequent new borders. The shape may however not be optimal (problem dependent) and other convex hulls can also be applied such as a hexagon or triangles. In general it holds that the shape determines the amount of over-approximation and the amount of computational load. More vertices generally allows for smaller evaluation boxes and thus less dependency and it allows for a tighter fit around the border of the reachable set. On the other hand more vertices means more evaluation boxes and generally a higher computational load. The effects of over-approximation might be severe if the incorrect shape is chosen but this is sometimes inevitable due to implementation issues. The optimization of shape selection is subject to further research. Even with the 'non-optimal' rectangular shapes the proposed method already shows encouraging results, which can only become better with more advanced shapes.

Future research will focus on applying the new theory to safe flight envelope determination, starting with the example by $\mathrm{Merz}^{29}$ of two adversarial vehicles in three dimensions, where the pursuer wishes to get within a certain distance of the evader and the reachable set is the set within which the pursuer can capture the evader.

\section{References}

\footnotetext{
${ }^{1}$ Ranter, H., "Airliner Accident Statistics 2006," Tech. rep., Aviation Safety Network, 2007.

${ }^{2}$ Lambregts, A., Nesemeier, G., Wilborn, J., and Newman, R., "Airplane Upsets: Old Problems, New Issues," AIAA Modeling and Simulation Technologies Conference and Exhibit, Honolulu, Hawaii, August 2008.

${ }^{3}$ Pandita, R., Chakraborty, A., Seiler, P., and Balas, G., "Reachability and Region of Attraction Analysis Applied to GTM Dynamic Flight Envelope Assessment," AIAA Guidance, Navigation, and Control Conference, Chicago, Illinois, USA, 10 - 13 August 2009.

${ }^{4}$ Ganguli, S., Ariyur, K. B., and Enns, D. F., "Region of Attraction with Performance Bounds," AIAA Guidance, Navigation, and Control Conference, Chicago, Illinois, USA, 2009.

${ }^{5}$ Osher, S. and Fedkiw, R., Level Set Methods and Dynamic Implicit Surface, Springer, 2003.

${ }^{6}$ Hwang, I., Stipanovic, D., and Tomlin, C., "Applications of polytopic approximations of reachable sets to linear dynamic games and a class of nonlinear systems," American Control Conference, 2003. Proceedings of the 2003, Vol. 6, June 2003, pp. $4613-4619$ vol.6.

${ }^{7}$ Shin, J., "Analysis of linear parameter varying system model based on reachable sets," Tech. Rep. CR 211231, NASA, Langley Research Center, 2001.

${ }^{8}$ Lygeros, J., "On reachability and minimum cost optimal control," Automatica, Vol. 40, No. 6, 2004, pp. 917 - 927.

${ }^{9}$ Cross, E. A. and Mitchell, I. M., "Level Set Methods for Computing Reachable Sets of Systems with Differential Algebraic Equation Dynamics," American Control Conference, Seattle, Washington, USA, 2008.

${ }^{10}$ Bayen, A. M., Mitchell, I. M., and Oishi, M. M. K., "Aircraft autolander safety through optimal control based reach set computation," Journal of Guidance, Control, and Dynamics, Vol. 30, No. 1, 2007, pp. 68 - 77.

${ }^{11}$ Mitchell, I. M., "The flexible, extensible and efficient toolbox of level set methods," Journal of Scientific Computing, Vol. 35, No. 2-3, 2008, pp. $300-329$.

${ }^{12}$ Lhommeau, M., Jaulin, L., and Hardouin, L., "Inner and outer approximation of capture basin using interval analysis," ICINCO-SPSMC, 2007, pp. 5-9.

${ }^{13}$ Moore, R., Interval Analysis, Prentice-Hall, Inc., 1966.

${ }^{14}$ van Kampen, E., Chu, Q., Mulder, J., and van Emden, M., "Nonlinear Aircraft Trim Using Interval Analysis," Proceeding of the AIAA Guidance, Navigation, and Control Conference and Exhibit, August 2007.
} 
${ }^{15}$ van Kampen, E., Zaal, P., de Weerdt, E., Chu, Q., and Mulder, J., "Optimization of Human Perception Modeling Using Interval Analysis," Jounal of Guidance, Control, and Dynamics, Vol. 33, 2010, pp. 42-52.

${ }^{16}$ van Kampen, E., de Weerdt, E., Chu, Q., and Mulder, J., "Aircraft Attitude Determination Using GPS and an Interval Integer Ambiguity Resolution Algorithm," AIAA Guidance, Navigation, and Control Conference, Chicago, Illinois, Aug. 10-13, 2009, 2009.

${ }^{17}$ de Weerdt, E., van Kampen, E., Chu, Q., and Mulder, J., "New Approach for Integer Ambiguity Resolution using Interval Analysis," ION Journal of Navigation, Vol. 55, No. 4, 2008.

${ }^{18}$ Filipe, N., de Weerdt, E., van Kampen, E., Chu, Q., and Mulder, J., "Terminal Area Energy Management Trajectory Optimization Using Interval Analysis," AIAA Guidance, Navigation, and Control Conference, Chicago, Illinois, Aug. 10-13, 2009, 2009.

${ }^{19}$ de Weerdt, E., Chu, Q., and Mulder, J., "Global Fuel Optimization for Constrained Spacecraft Formation Rotations," AIAA Guidance, Navigation, and Control Conference, 2009.

${ }^{20}$ Kurzhanski, A. and Varaiya, P., "Dynamic optimization for reachibility problems," Journal of Optimization Theory and Applications, Vol. 108, No. 2, 2001, pp. $227-251$.

${ }^{21}$ Khrustalev, M., "Exact description of reachable sets and global optimality conditions," Avtomatika $i$ Telemekhanika, Vol. 5, 1988, pp. $62-70$.

${ }^{22}$ Hickey, T., Ju, Q., and van Emden, M., "Interval Arithmetic: from Principles to Implementation," Journal of the Association for Computing Machinery, Vol. 48, No. 5, 2001, pp. 1038-1068.

${ }^{23}$ Moore, R., Methods and Applications of Interval Analysis, SIAM, Philadelphia, PA, 1979.

${ }^{24}$ Makino, K. and Berz, M., "Higher Order Verified Inclusions of Multidimensional Systems by Taylor Models," Nonlinear Analysis, Vol. 47, 2001, pp. 3503-3514.

${ }^{25}$ Makino, K. and Berz, M., "Taylor Models and Other Validated Functional Inclusion Functions," International Journal of Pure and Applied Mathematics, Vol. 4, No. 4, 2003, pp. 379-456.

${ }^{26}$ Corliss, G., editor, Taylor Series Models in Deterministic Global Optimization. Springer-Verlag, 2000.

${ }^{27}$ Berz, M. and Hoffstatter, G., "Computation and Application of Taylor Polynomials with Interval RemainderBounds," Reliable Computing, Vol. 4, 1998, pp. 83-97.

28 "INTLAB - INTerval LABoratory," http://www.ti3.tu-harburg.de/rump/intlab/, Institute for Reliable Computing,Hamburg University of Technology,Schwarzenbergstr.95.

${ }^{29}$ Merz, A. W., "The game of two identical cars," Journal of Optimization Theory and Applications, Vol. 9, No. 5, 1972, pp. $324-343$. 